\title{
METODOLOGÍA ACTIVA “PROJECT BASED LEARNING” COOPERANDO CON LAS TICS Y LA ADAPTACIÓN DE SU EVALUACIÓN
}

Active methodology "Project Based Learning" cooperating with the ITCs and adapting their evaluation

Active metodologia "Project Based Learning "cooperar com as TICs e adaptação da sua avaliação

\section{Leyla Angélica Sandoval Hamón (1)}

\section{Ricardo Olmos Albacete (2)}

(1) Universidad Autónoma de Madrid (UAM). Teléfono: +34914975518. Correo electrónico: angelica.sandoval@uam.es

(2) Universidad Autónoma de Madrid (UAM). Correo electrónico: ricardo.olmos@uam.es

\section{Resumen}

El EEES propicia el cambio en la enseñanza-aprendizaje, donde los estudiantes tengan un papel más activo en su proceso de educación. Por ello, el principal objetivo de este trabajo es presentar una propuesta alternativa a la tradicional centrada en la actividad autónoma del estudiante, que les permita adquirir y profundizar una mayor diversidad de conocimientos y competencias. La puesta en marcha de esta propuesta se realizó en la asignatura Seminario de administración de empresas y entorno socio político, empleando la metodología del aprendizaje basado en proyectos integrando TICs y adaptando la evaluación (incorporando rubricas, análisis psicométricos de las pruebas de conocimientos, etc.) a esta forma de plantear la asignatura. La experiencia concluyó con la observación, recogida de trabajos, pruebas de conocimientos (análisis de los mismos), y la cumplimentación de un cuestionario y encuesta oficial por parte de estudiantes para valorar la actividad y el desarrollo de sus competencias.

Palabras clave: Aprendizaje Basado en Proyectos; Trabajo en grupos; TICs; Evaluación

\begin{abstract}
In the European space of higher education, it favors the change in teaching-learning, where students have a more active role in their education process. Therefore, the main Metodología activa "project based learning" cooperando con las TICS y la adaptación de su evaluación
\end{abstract}


objective of this work is to present an alternative proposal to the traditional one focused on the autonomous activity of the student, which allows them to acquire and deepen with a greater diversity of knowledge and skills. The implementation of this proposal was carried out in the Seminar of business administration and socio-political environment, using the methodology of Project-based learning integrating ICTs and adapting the evaluation (incorporating rubrics, psychometric tests of knowledge tests, etc.) to this way of raising the subject. The experiment concluded with the observation, collection of works and knowledge tests, analysis of the same, and the completion of a questionnaire and official survey by students to assess the activity and the development of their skills.

Keywords: Project Based Learning; Group Works; ICTs; Evaluation

\section{Resumo}

O Espaço Europeu do Ensino Superior (EEES) propicia a mudança no ensinoaprendizagem, onde os estudantes têm um papel mais ativo no seu processo de formação. Como tal, o principal objetivo deste trabalho é apresentar uma proposta alternativa à tradicional, centrada na actividade autónoma do estudante, permitindo-lhes adquirir e aprofundar com uma maior diversidade de conhecimentos e competências. A implementação desta proposta foi feita na Unidade Curricular de Seminário de Administração de Empresas e Ambiente Sócio-Político, utilizando a metodologia de aprendizagem baseada em projetos, integrando as TIC e adaptando a avaliação (incorporando rubricas, análise psicométrica dos testes de conhecimento, etc.) de forma a realizar a Unidade Curricular. A experiência terminou com a observação, recolha de trabalhos e testes de conhecimento, análise dos mesmos, e a execução de um questionário e inquéritos por parte dos estudantes para avaliar a actividade e o desenvolvimento de suas competências.

Palavras-chave: Projeto de Aprendizagem Baseada; O trabalho em grupo; TICs; Avaliação

\section{Introducción}

Este trabajo se centra en el análisis de una experiencia docente al emplear una metodología alternativa a la tradicional, el aprendizaje basado en proyectos(ABP) (March, 2006; Hmelo-Silver \& Barrows, 2006; Bransford et al., 2000), propiciando al 
mismo tiempo, el uso de las tecnologías de la información y de la comunicación (TIC). Además, se ha realizado una adaptación (metodología $\mathrm{ABP}$ ) en cómo se evalúa la asignatura, al considerar que la evaluación es "el arma más poderosa que tienen los profesores para influir en el modo en el que los estudiantes responden a los cursos y se comportan como alumnos" (Gibbs, 2003, p. 61).

\section{Contextualización}

Titulación/Institución superior: Economía/Universidad Autónoma de Madrid

Curso: Cuarto grado. 2016-2017.

Asignatura: Seminario de administración de empresas y entorno socio-político.

Estudiantes: 35

N ${ }^{\circ}$ Créditos: 6 (150 horas: 88 teóricas y 62 prácticas)

Este seminario pretende acercar al estudiante a la realidad del funcionamiento del sistema económico y del comportamiento de las empresas.

Número de temas: 10 .

\section{Diseño y desarrollo}

Plan de trabajo:

Al comienzo del curso, el equipo docente se reunió y planificó todas las sesiones de clase compartiéndolas con los estudiantes. Un resumen, del plan de trabajo docente en tabla 1.

Tabla 1.

Metodologías/evaluación y tareas

\begin{tabular}{lll}
\hline Fase & Descripción & Tareas \\
\hline 1 & ABP & 1.1 Elaboración de las propuestas \\
& & 1.2 Planificación y seguimiento para hacer los proyectos \\
& & 1.3 Comprobación de resultados \\
& & 1.4 Evaluación (formativa y compartida) \\
\hline 2 & TICs & 2.1 Identificar recursos tecnológicos.(Listado) \\
& & 2.2 Compartir recursos TICs y apoyo a estudiantes en empleo de TICs. \\
\hline 3 & Evaluación & 3.1 Elaboración de rúbricas para los proyectos. \\
& & 3.2 Base de datos de preguntas breves sobre temas de la asignatura y realizarlas \\
& & con Kahoot. \\
& & 3.3 Elaboración de dos test (revisados por pares y análisis psicométrico de los \\
& & tests (Test Analysis Program, TAP) \\
& &
\end{tabular}


El eje principal del trabajo de los estudiantes en la asignatura consistió en: desarrollar dos proyectos por grupos (5 estudiantes), uno relacionado con el análisis de un sector de actividad y otro, con el análisis de una empresa del sector, empleando TICs. El desarrollo de estos proyectos consideraba tener como referencia los contenidos de la asignatura. El resultado de los proyectos se vio reflejado principalmente en informes finales, presentaciones orales y en dos test sobre los contenidos de la asignatura.

En línea con la metodología activa, se desarrolló un sistema de evaluación, donde los estudiantes: a) pudieran tener un rápido diagnóstico de su progreso en cada tema (a través la plataforma educativa de kahoot donde se realizaban preguntas concretas sobre temas de la asignatura), b) tuvieran con antelación los criterios de evaluación de las actividades grupales (de los dos proyectos) para que, una vez realizados los informes y las presentaciones, reconozcan sus fortalezas y debilidades y c) pudieran tener un análisis agregado(TAP) de las pruebas (en particular, se analizaron dos test con 32 preguntas cada una y 4 opciones de respuesta y con penalización por errores)

\section{Evaluación y resultados}

La evaluación estuvo compuesta por:

- Rubricas para la realización de informes y de presentaciones orales de proyectos. Estas rúbricas consideraban: búsqueda de información, organización, contenido, presentación, etc.

- Preguntas breves sobre los temas de la asignatura con Kahoot. El grado de participación fue alto(70\%), e impulsó a los estudiantes a mejorar sus capacidades y, con ello, resultados.

- Dos pruebas de conocimientos antes mencionadas, tipo test donde se incluían contenidos de toda la asignatura.

Análisis psicométrico de los dos test (permiten tener el análisis agregado de las pruebas, con el que se averigua las fiabilidades y las propiedades psicométricas de las preguntas como evolución a las buenas prácticas desarrolladas en la elaboración de test, al ser revisados por pares). En las figuras de abajo se muestra la distribución de aciertos en ambos exámenes tipo test. 
Prueba de conocimientos 1

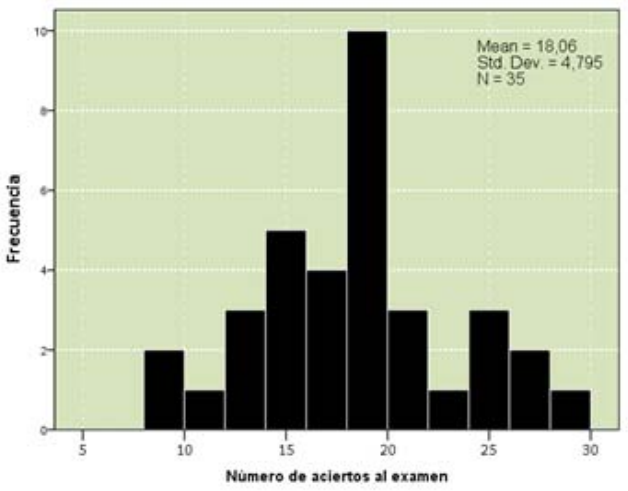

Figura 1.

Histograma de resultados prueba 1.
Prueba de conocimientos 2

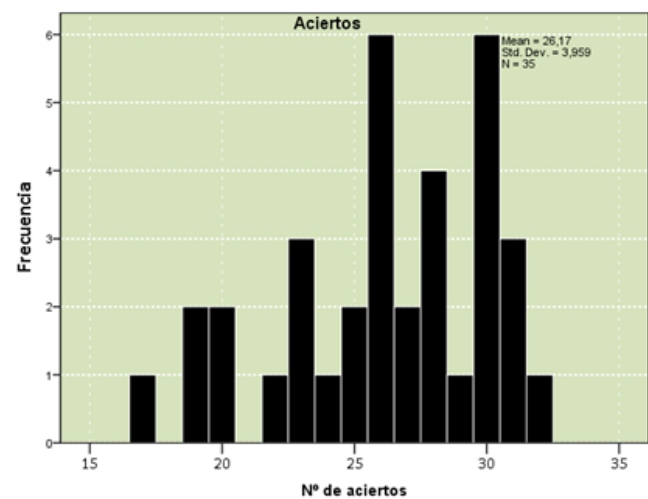

Figura 2.

Histograma de resultados prueba 2.

Resultados test 1.

La media de aciertos en el examen de 32 preguntas fue de 18,06 (desviación típica de 4,80). El examen tuvo dificultad media (no fue ni excesivamente fácil, ni excesivamente difícil), por lo que se adaptó adecuadamente al nivel de conocimientos de los estudiantes de la asignatura. El coeficiente de fiabilidad de este examen, además, fue aceptable ( $\alpha$ de Cronbach $=0,72$ ), más elevado que las fiabilidades medias encontradas en los exámenes de la universidad y por encima de 0,70 , criterio arbitrario estándar para valorar que un examen es fiable.

\section{Resultados test 2.}

La media de aciertos en el examen de 32 preguntas fue de 26,17 (desviación típica de 3,90 ), por lo que la prueba de conocimientos 2 tuvo dificultad media-baja (comparado con el examen 1 fue un poco más fácil). En este caso, el coeficiente de fiabilidad $\alpha$ de Cronbach fue aún más elevado, 0,76, lo que muestra que el test evaluó los conocimientos de los estudiantes con bastante precisión.

\section{Conclusiones}

En el contexto de transformación de la docencia para favorecer el aprendizaje activo de los estudiantes, este proyecto ha introducido, en un curso concreto, una metodología basado en proyectos e integrando las TICS), adecuando los sistemas de evaluación mediante rubricas (criterios para las entregas de proyectos), preguntas con aplicaciones móviles (sobre contenido puntual) y análisis de dos exámenes tipo test. 
La experiencia en la asignatura ha resultado positiva, porque se ha avanzado en que los estudiantes se encuentren mejor preparados en competencias adquiridas, no solo en conocimientos teóricos sobre las organizaciones, sino en que sean capaces de actuar con respecto a ellas de forma crítica e integren las TICS, en sus análisis y presentaciones, de manera práctica. Una evidencia de este avance ha sido la mejora en las calificaciones y, además, por la satisfacción mostrada por éstos con la asignatura (recogida en la encuesta oficial realizada con puntuaciones superiores a la media de las otras asignaturas) y que se muesta a partir de la siguiente nube de palabras:

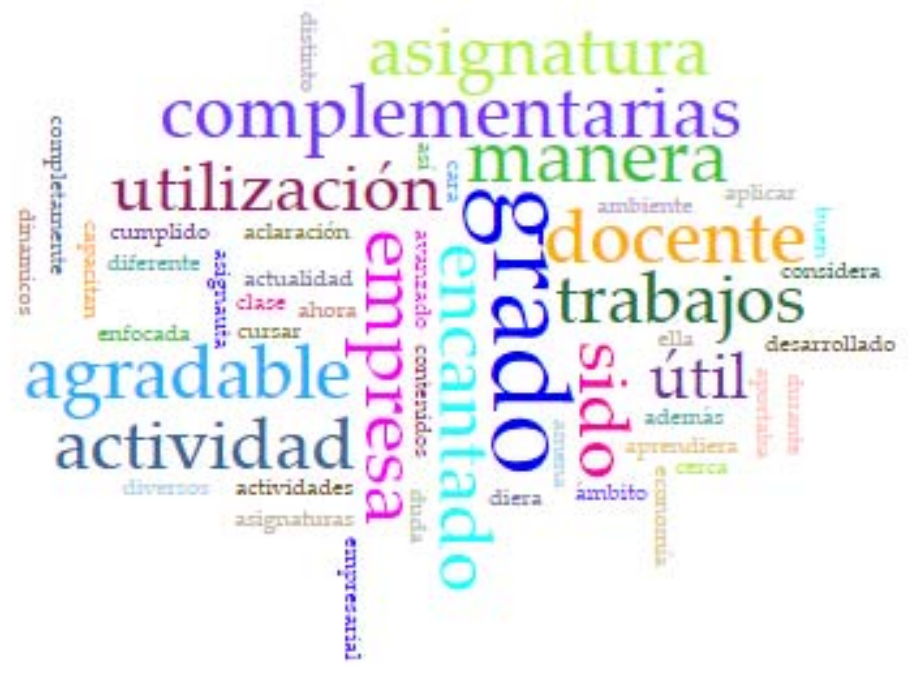

Figura 3.

Nube de palabras a partir del corpus formado por los comentarios de los estudiantes en las encuestas oficiales

\section{Referencias}

Bransford, J. D., Brown, A. L., \& Cocking, R. R. (2000). How People Learn. Brain, Mind, Experience, and School. Washington: National Academies Press.

Gibbs, G. (2003). Uso estratégico de la educación en el aprendizaje. Evaluar en la universidad: problemas y nuevos enfoques (pp. 61-74). Madrid: Narcea.

Hmelo-Silver, C. E., \& Barrows, H. S. (2006) Goals and strategies of a problem-based learning facilitator, Interdisciplinary Journal of Problem-based Learning, 1, 2039.

March, A. F. (2006). Metodologías activas para la formación de competencias. Educatio siglo XXI, 24, 35-56. 